\title{
Fast electron generation in cones with ultra-intense laser pulses
}

A.J. Mackinnon, L. VanWoerkom, K.U. Akli, T. Bartal, F.N. Beg, S. Chawla, C. Chen, E. Chowdhury, R.R. Freeman, D. Hey, M.H. Key, J.A. King, A. Link, A.G. MacPhee, D. Offermann, V. Ovchinnikov, P.K. Patel, D.W. Schumacher, R.B. Stephens, Y. Tsui

December 10, 2007

Physics of Plasmas 
This document was prepared as an account of work sponsored by an agency of the United States government. Neither the United States government nor Lawrence Livermore National Security, LLC, nor any of their employees makes any warranty, expressed or implied, or assumes any legal liability or responsibility for the accuracy, completeness, or usefulness of any information, apparatus, product, or process disclosed, or represents that its use would not infringe privately owned rights. Reference herein to any specific commercial product, process, or service by trade name, trademark, manufacturer, or otherwise does not necessarily constitute or imply its endorsement, recommendation, or favoring by the United States government or Lawrence Livermore National Security, LLC. The views and opinions of authors expressed herein do not necessarily state or reflect those of the United States government or Lawrence Livermore National Security, LLC, and shall not be used for advertising or product endorsement purposes. 


\title{
Fast electron generation in cones with ultra-intense laser pulses
}

L. Van Woerkom ${ }^{1}$, K.U. Akli ${ }^{2}$, T. Bartal ${ }^{3}$, F.N. Beg ${ }^{3}$, S. Chawla ${ }^{3}$, C. Chen ${ }^{4}$, E. Chowdhury ${ }^{1}$, R.R. Freeman ${ }^{1,3,5}$, D. Hey ${ }^{5,6}$, M.H. Key ${ }^{6}$, J.A. King ${ }^{3}$, A. Link ${ }^{1}$, T. Ma ${ }^{3}$, A.J. MacKinnon ${ }^{6}$, A. MacPhee $^{6}$, D. Offermann ${ }^{1}$, V. Ovchinnikov ${ }^{1}$, P.K Patel ${ }^{6}$, D.W. Schumacher ${ }^{1}$, R.B. Stephens ${ }^{2}$, Y. Tsui $^{7}$

${ }^{1}$ Department of Physics, The Ohio State University, Columbus, OH 43210

${ }^{2}$ General Atomics, San Diego, CA 92186

${ }^{3}$ Department of Mechanical and Aerospace Engineering, University of California San Diego, La Jolla, CA 92093

${ }^{4}$ Department of Physics, Massachusetts Institute of Technology, Cambridge, MA 02139-4307

${ }^{5}$ Department of Applied Science, University of California, Davis, Livermore, CA 94550, USA

${ }^{6}$ University of California, Lawrence Livermore National Laboratory, Livermore CA 94550, USA

${ }^{7}$ Department of Electrical \& Computer Engineering, University of Alberta Edmonton, Alberta Canada T6G $2 \mathrm{~V} 4$

\begin{abstract}
Experimental results from copper cones irradiated with ultra-intense laser light are presented. Spatial images and total yields of $\mathrm{Cu} \mathrm{K} \mathrm{K}_{\alpha}$ fluorescence were measured as a function of the laser focusing properties. The fluorescence emission extends into the cone approximately $300 \mu \mathrm{m}$ from the cone tip and cannot be explained by ray tracing including cone wall absorption. In addition the total fluorescence yield from cones is an order of magnitude higher than for equivalent mass foil targets. Indications are that the physics of the laser cone interaction is dominated by preplasma created from the long duration, low energy pre-pulse from the laser.
\end{abstract}




\section{Introduction}

While the basic idea for Fast Ignition (FI) driven Inertial Confinement Fusion (ICF) is straightforward in concept ${ }^{1}$, the actual realization of the technique, and particularly the injection of igniter laser, relies upon a fundamental understanding of many complex physics issues, ranging from laser-plasma interactions at high fields to high current charge transport through dense plasmas. For example, the mechanisms governing laser light conversion into energetic electrons (in number, energy and direction) must be measured and understood in order to provide accurate scaling for laser driver development. The design of the fuel assembly in the target must take into account the need for protecting the igniter laser path from the implosion plasma, thus all but demanding the inclusion of a cone within the target. ${ }^{2}$ Notably both the effect of the cone on the implosion as well as the implosion on the cone must be understood ${ }^{3}$, the subsequent interaction with the igniter laser must be modeled in detail. Finally, the physics of the electron transport process from the generation point near the cone tip to the compressed fuel core needs full characterization. ${ }^{4}$

Because it is likely that initial large scale FI laser systems will consist of multiplexing multiple laser beams with modest f-numbers, techniques for combining beams in a coherent fashion to deliver the laser light energy onto the fuel in a diameter of approximately 30 to $40 \mu \mathrm{m}$ will be needed. ${ }^{5}$ Until such techniques are developed and demonstrated, cones will likely be called upon to provide assistance in concentrating the ignition laser light onto the fuel. There have been several recent studies on the interaction of intense light with cones and cone-like structures. These include geometric effects via simulations and experiments. ${ }^{6-14}$

This paper discusses our recent explorations in laser-cone interactions. Section II describes the experimental setup and relevant parameters. Section III presents experimental results for $\mathrm{K}_{\alpha}$ 
yields, variable focusing conditions in cones, ray tracing and the effects of pre-pulse. A discussion of dominant physics issues is given in section IV. Section V discusses the implications for fast ignition while section VI offers conclusions.

\section{Experimental Setup}

In an attempt to gain insight into the optical guiding of cones for fast ignition, we have performed a series of experiments in which the focal properties of the laser were varied for a given cone geometry. These measurements employed a low f/number input laser beam (f/3) focused into a $15^{\circ}$ half-angle cone. These experiments were specifically designed to give us experience in understanding optical guiding effects in cones including realistic laser pre-pulse.

All of the experiments described in this paper were performed on the Titan Laser at the Jupiter Laser Facility at Lawrence Livermore National Laboratory. The laser operated at $1.054 \mathrm{~mm}$ with a pulse duration of about $600 \mathrm{fs}$ and a maximum energy of $150 \mathrm{~J}$ per shot, yielding a peak focused intensity of about $10^{20} \mathrm{~W} / \mathrm{cm}^{2}$. The short pulse was preceded by a $\sim 3 \mathrm{~ns}$ long ASEgenerated pre-pulse with a typical energy of about $14 \mathrm{~mJ}$. This $10^{4}: 1$ energy contrast leads to a $10^{8}: 1$ intensity contrast on target, which gives a pre-pulse intensity of about $10^{12-13} \mathrm{~W} / \mathrm{cm}^{2}$ for the highest energy shots. We employed two types of targets for these studies: (a) $25 \mu \mathrm{m}$ thick copper foils irradiated with s-polarization and (b) copper cones with $25 \mu \mathrm{m}$ wall thickness (equivalent mass to that of $1 \mathrm{~mm}$ square foils).

\section{Results}

\section{A. Cone Wall Interactions}

The first step in understanding the role of intense lasers interacting with cones is to study a surrogate for the cone wall: flat foils irradiated at oblique angles of incidence. The laser was focused onto $0.5 \mathrm{~mm}$ square, 25 micron thick copper foils at $75^{\circ}$ angle of incidence, s-polarized 
with respect to the target normal. Three measurements were made: 1) reflectivity relative to near normal incidence at $\left.28^{\circ}, 2\right)$ total $\mathrm{K}_{\alpha}$ fluorescence yield relative to near normal incidence at $28^{\circ}$, and 3) 2-dimensional images of $\mathrm{Cu} \mathrm{K}_{\alpha}$ fluorescence from both sides of the foil.

Reflectivity measurements were made by collecting the specular reflection from the foil targets on a piece of Spectralon ${ }^{\mathrm{TM}}$ scattering material and relaying the image to a calibrated CCD camera. Although absolute numbers for the system are not yet available, the relative reflectivity between near oblique and normal incidence was found to be substantial with the reflectivity for $75^{\circ}$ about 20 times that from the near normal $28^{\circ}$ incident case. In addition the absolute yield of $\mathrm{K}_{\alpha}$ fluorescence was measured using a Highly Oriented Pyrolytic Graphite (HOPG) spectrometer absolutely calibrated with a single hit CCD (SHCCD) spectrometer: the yield from oblique foils was lower by an order of magnitude with respect to near normal foils.

Two dimensional spatial images of the $\mathrm{Cu} \mathrm{K}_{\alpha}$ fluorescence were obtained using spherically curved Bragg crystal imagers. ${ }^{15}$ In particular the $25 \mu \mathrm{m}$ thick $\mathrm{Cu}$ foils were used with transverse dimensions of $0.5 \mathrm{~mm}$. Measurements at near normal to the target normal showed a bright $\mathrm{K}_{\alpha}$ emission spot roughly in the center of the foil with symmetric weak intensity enhancements at the four corners. The corner fluorescence is a result of small numbers of energetic electrons dispersing laterally and depositing their energy at the corner tips. In an attempt to observe surface electron transport, similar foils were irradiated at $75^{\circ}$ with respect to the target normal. Any increase and/or asymmetry in the transverse velocity distribution were expected to be revealed as an asymmetry in the edge fluorescence. Figure 1 shows typical data from these shots. Unexpectedly, the only sign of enhanced $\mathrm{K}_{\alpha}$ emission on the foil are on the side opposed to the laser propagation direction. Thus, we observe no strong evidence for the transport of energetic electrons parallel to the target surface. It is important to note that such electrons could not have 
been overlooked: The foils were thin enough to observe fluorescence throughout its bulk, and surface fields build up too quickly to allow a significant number of electrons to escape without fluorescing. Other groups have measured beamed electrons in vacuum and suggested that this is due to surface transport. ${ }^{14,16,17}$ However, the total number of electrons escaping into vacuum in these experiments is very small and the correlation of those electrons to the internal distribution is weak at best. ${ }^{18}$ A simple argument sets the physical limits. To estimate the number of $1 \mathrm{MeV}$ electrons that can escape, model the target as a $1 \mathrm{~mm}$ diameter sphere. Target charging limits the total number of $1 \mathrm{MeV}$ electrons escaping into vacuum to approximately $10^{11}$. The total number of hot electrons generated in the target is approximately $10^{13}$ electrons based on $30 \%$ coupling of laser light to hot electrons (and further reduced by an order of magnitude for oblique incidence). Thus, fewer than $1 \%$ (at most) of the generated electrons escape. In terms of fast ignition there is no relevant energy carried by such electrons.

Our studies of oblique compared to near-normal irradiation of foils reveal: 1) the reflectivity is much higher, 2) the $K_{\alpha}$ fluorescence yield is lower by an order of magnitude, and 3) there is no obvious transport of electrons inside the target parallel to the surface in the laser direction. These conclusions relate to s-polarization and we are currently preparing a similar study for $\mathrm{p}$ polarization where we anticipate even less reflection at oblique incidence.

\section{B. Defocused Illumination of Cones}

Laser-cone interactions were studied using both absolute $\mathrm{K}_{\alpha}$ fluorescence yield and 2dimensional $\mathrm{K}_{\alpha}$ imaging. Utilizing the same $\mathrm{Cu} \mathrm{K}_{\alpha}$ Bragg imager described above, measurements were made on $\mathrm{Cu}$ cones with a variety of focusing conditions. In all cases the laser focus was aligned with the cone axis with the laser polarization axis approximately perpendicular to the viewing direction. The cones were constructed of $25 \mu \mathrm{m}$ thick copper with 
an internal cone tip diameter of $30 \mu \mathrm{m}$ and an overall length of about $800 \mu \mathrm{m}$. The cones were supported by an end flange attached to two $25 \mu \mathrm{m}$ titanium stalks which were secured $\sim 3 \mathrm{~mm}$ above a $3 \mathrm{~mm}$ diameter aluminum mounting post.

Figure 2 shows $\mathrm{K}_{\alpha}$ fluorescence images for four different defocusing conditions: (a) tight focusing directly on the cone tip, (b) focusing the laser approximately $400 \mu \mathrm{m}$ beyond the cone tip, (c) focusing the laser about $400 \mu \mathrm{m}$ behind the cone tip, and (d) focusing the laser by $800 \mu \mathrm{m}$ beyond the cone tip, which essentially fills the cone with the laser beam. The $\mathrm{K}_{\alpha}$ fluorescence extends from the cone tip to about $300 \mu \mathrm{m}$ up the cone for all focusing conditions. The grid cone structure superimposed on the fluorescence emission was derived by taking the manufactured cone dimensions and projecting the geometry through the imaging system to the detector plane. Due to the slight visibility of the end flange in the $\mathrm{K}_{\alpha}$ image, it is possible to uniquely orient the cone with respect to the fluorescent image. Below each image is a line out along the cone axis and averaged over several pixels. Integrating over more transverse pixels or even entire transverse segments of the fluorescence does not change the essential feature of the line outs. Note that the images for all three defocusing conditions (b-d) are very similar to each other as well as to the tight focus shot in (a). As the focus is moved away from the cone tip there is an indication that the fluorescence peak is shifting slightly away from the cone tip as well, although nothing definitive can be said without more data. Figure 3 shows the overall common characteristics of the fluorescence line outs. First, there is a steep rise in $50 \mu \mathrm{m}$ at the cone tip which is followed by a broad extent of about $140 \mu \mathrm{m}$. Finally, all the curves exhibit an exponential decay with a scale length of about $140 \mu \mathrm{m}$. (It is interesting to note the decay scale length along the cone wall is equal to the scale length for $\mathrm{K}_{\alpha}$ fluorescence observed in cone wires and nail targets due to electric inhibition from fields generated by return currents interacting with 
the cold material.) The absolute fluorescence yields for all focusing conditions into the cones (when normalized for laser energy) were nearly identical, and all were an order of magnitude larger than equivalent shots on flat foils of identical masses

In summary, in our cone shots, we found that under a wide variety of differing focal conditions, we not only recorded very nearly identical total $\mathrm{K}_{\alpha}$ yields, but nearly identical $\mathrm{K}_{\alpha}$ fluorescence images as well: these images extended about $300 \mu \mathrm{m}$ up the cone from the tip.

\section{Ray Tracing}

The first step in developing a physical picture for the observed similarity in the $\mathrm{K}_{\alpha}$ fluorescence for all cone shots was to use realistic optical ray tracing. The optical design program FRED $^{\mathrm{TM}}$ was used to study optical guiding for each of the focusing conditions described above. Fig. 4 shows an example of optical guiding for the case of the 400 micron focus beyond the cone tip which corresponds to the data shown in figure 2(b). Highlighted in black is a sample ray showing the reflections, which shows that after the first reflection, subsequent bounces have an angle of incidence less than $45^{\circ}$ from the surface normal. This is important because absorption for small angles of incidence with respect to the wall normal will be large. Thus, while the ray tracing demonstrates optical guiding, a more complete picture must include cone-wall absorption for a realistic picture of interactions near the tip. We used a simple description of the absorption, based on high intensity absorption measurements at LLNL who measured $\sim 60-65 \%$ absorption at $10^{20} \mathrm{~W} / \mathrm{cm}^{2}$ for $6^{\circ}$ and $45^{\circ}$ angles of incidence with $\mathrm{p}$ polarization. ${ }^{19}$ Our model combines this information with the observation of $20 x$ enhanced specular reflection at $75^{\circ}$ (albeit for s-polarization) to construct the angle-dependent absorption fraction as a function of incidence angle fixed at $65 \%$ for all angles less than $55^{\circ}$ followed by a linear decrease to no absorption for glancing angles. The results of ray tracing using FRED $^{\mathrm{TM}}$ 
including absorption are given in figure 5 where the graph shows the absorption in the cone wall as a function of distance from the cone tip. Note that the cone-wall absorption becomes significant only within $50 \mu \mathrm{m}$ of the cone tip.

Although the details of the absorption values used in the ray tracing are not necessarily complete, the basic observations remain the same. The only vital feature is an increased absorption for angles of incidence less than about $55^{\circ}$ with respect to the surface normal. Thus, significant $\mathrm{K}_{\alpha}$ fluorescence emission from the $\mathrm{Cu}$ cone wall would only be expected within 50 $\mu \mathrm{m}$ away from the cone tip if there were no additional processes involved. However, the observed $\mathrm{K}_{\alpha}$ images show strong emission for varied focal geometries out to $300 \mu \mathrm{m}$ from the cone tip. This provides strong evidence that additional physics beyond simple cone-wall absorption is important in understanding the interaction of intense laser light with the cone.

\section{The Role of Laser Pre-pulse}

The previous discussion using ray tracing relied on an ideal ultra-intense laser pulse interacting with the target material. In reality all short pulse lasers have some amount of prepulse which deposits non-negligible energy on the target up to nanoseconds before the ultraintense short pulse arrives. As mentioned in Section II the Titan laser produces a typical prepulse energy of about $14 \mathrm{~mJ}$ spread over about $3 \mathrm{~ns}$ before the main short pulse.

The effect this pre-pulse has on pre-heating the cone material can be estimated using information from our experiments on flat foils with interferometric side probing. Such measurements on Titan yielded a pre-plasma with an axial scale length of about $100 \mu \mathrm{m}$ and a lateral scale length of about $50 \mu \mathrm{m}$ for a density out to $\sim 10^{18} \mathrm{cc}^{-1}$. Importantly, recent work by Baton et al., ${ }^{20}$ using side-on shadowgraphy through a cone-like structure with an open slit near the tip, have shown the presence of pre-plasma over $100 \mu \mathrm{m}$ away from the cone tip for similar 
pre-pulse levels. Recent calculations of the pre-pulse for a cone geometry similar that used in our experiments indicate that the pre-plasma extends even further in a cone geometry than from a flat target. ${ }^{21}$ The critical density for one micron light is pushed out to about $60 \mu \mathrm{m}$ from the cone tip with densities greater than $10^{20} \mathrm{cc}^{-1}$ occurring out to a few hundred $\mathrm{mm}$. We estimate that these are the conditions into which the high intensity short pulse is focused into the cones.

\section{Discussion}

To obtain a more complete picture of the interaction physics inside the cone we combine ray tracing, wall absorption and the effects of pre-plasma. Lacking a complete, self-consistent model, we use physical arguments to guide our interpretation of the data. The critical observations for the pattern of $\mathrm{Cu} \mathrm{K} \mathrm{K}_{\alpha}$ fluorescence are: 1) it is invariant with respect to the focal plane of the laser, 2) it extends further up the cone than predicted by ray tracing, and 3) its extent is larger than the radial spreading for equivalent mass and thickness flat foils. Finally we also observe that the total fluorescence yield is significantly higher than flat foils of similar mass and thickness. The increased total yield indicates a higher laser light absorption fraction.

In the absence of any pre-plasma, either inside or outside the cone, the cone represents an ideal refluxing target. That is, without any additional conductive pathways, the electrons will be roughly bound to individual cone walls via strong electrostatic sheath fields on both the inside and outside of the cone walls, similar in principle to a thin flat foil. Laser absorption creates hot electrons on the inner surface of the cone (or at least near it) and they subsequently traverse through the cone wall into the vacuum outside the cone where they set up strong sheath electric fields. These fields drive subsequent electrons back into the cone wall and hence into the cone interior. In the absence of any plasma in the cone these refluxed electrons set up an interior sheath field, and electrons reflux back into the cone wall, effectively interacting with the cone tip 
region out to about $50 \mu \mathrm{m}$. However, with pre-plasma filling in the cone, the interior sheath fields are essentially shorted, and the first refluxing fast electrons from one interior cone wall can diffuse through to the opposite wall as long as the fast electron density is less than the interior plasma density. The substantial increased spreading of the $K_{\alpha}$ fluorescence, independent of where laser is focused, is due to the presence of this preformed plasma in the cone that makes the final $300 \mu \mathrm{m}$ behave like a region of plasma in which the hot electrons can freely diffuse, even thought the laser absorption occurs in the last $50 \mu \mathrm{m}$. The density of energetic electrons in the cone out to $300 \mu \mathrm{m}$ can be estimated using the same arguments made above regarding the electrons escaping into vacuum, giving $\mathrm{n}_{\text {fast }} \sim 10^{20} \mathrm{cc}^{-1}$. These electrons excite fluorescence when their paths intersect the dense wall region so emission occurs everywhere the electrons can freely travel. Since the $\mathrm{K}_{\alpha}$ fluorescence imager is a time integrated measurement, it is impossible to tell when the electrons interacted with a given part of the cone wall; it is sensitive only to the average fast electron density over the course of the fast electron lifetime.

\section{Implications for Fast Ignition}

The previous discussion underscores the importance of understanding the physics of intense laser light interaction with cones for realistic systems in the presence of pre-plasma. For FI, a large number of electrons with energies between 1.5 and $2.5 \mathrm{MeV}$ directed toward the compressed fuel core with a small divergence angle must be produced. Although we observe large total $\mathrm{K}_{\alpha}$ yields, our diagnostic records only the total fluorescence integrated over space and time. We have shown with flat foil surrogates that there is no significant electron flow along the metal surface, so the only way to generate the necessary electrons is to get the laser light to the cone tip. Observations of increased fluorescence in cones is consistent with ray tracing models that show multiple bounces as light nears the cone tip, and have little to do with where the 
energy is deposited. Imaging the hot-electron generated fluorescence in cones shows some transport along the length of the cone - as much away from the tip as toward it. We suggest that pre-pulse generated plasmas, shown here to be more extensive in the cone geometry, are allowing this. More information is needed to discriminate the generation of electrons at the tip from the rest of the cone.

In terms of large scale FI one major question is: What is the role of the pre-plasma in the presence of a mid $10^{20} \mathrm{~W} / \mathrm{cm}^{2}$ laser field for which the light pressure may be high enough to sweep up a significant amount of pre-plasma? If large scale igniter lasers maintain an energy contrast of about $10^{4}: 1$, multi-kJ igniter lasers could produce up to a Joule of energy a few nanoseconds before the main short pulse beam. Current experiments are being fielded to address density profile steepening and subsequent fast electron generation. However, at this point, it remains on open question whether the full igniter beam will be able to sweep up enough preplasma to permit a clean interaction with the cone tip.

\section{Conclusion}

In summary we have studied the interaction of intense laser light with FI relevant cones. Our measurements are consistent with optical guiding in the cone, but we fail to detect the presence of significant electron guiding along the surface or in the cone wall. While the cones provide effective light guiding, the measured $\mathrm{Cu} \mathrm{K}_{\alpha}$ fluorescence yields are most probably dominated by effects of pre-plasma filling the cone interior. We propose that the plasma filling of the cone provides a means for the fast electrons to be dispersed and scattered effectively throughout the cone out to $300 \mathrm{~mm}$ away from the tip. It would appear that the even greater pre-pulse energies on full-scale FI ignition lasers will enhance this effect. Further efforts are needed in order to develop realistic point design criteria for fast ignition. 


\section{ACKNOWLEDGMENTS}

This work was supported by the Office of Fusion Energy Science, the Fusion Science Center.

This work was also performed under the auspices of the U.S. Department of Energy by

Lawrence Livermore National Laboratory under Contract DE-AC52-07NA27344. 
M. J. Tabak, Hammer, M. E. Glinsky, W. L. Kruer, S. C. Wilks, J. Woodworth, E. M. Campbell, M. D. Perry, and R. J. Mason, PHYSICS OF PLASMAS 1, 1626 (1994).

S. Hatchett and M. Tabak, in 30th Anomalous Absorption Conference, Ocean City, MD, (2000).

S. P. Hatchett, D. Clark, M. Tabak, R. E. Turner, C. Stoeckl, R. B. Stephens, H. Shiraga, and K. Tanaka, Fusion Science and Technology 49, 327 (2006).

R. R. Freeman, D. Batani, S. Baton, M. H. Key, and R. Stephens, Fusion Science \& Technology 49, 297 (2006).

S. Atzeni, A. Schiavi, and C. Bellei, Physics of Plasmas 14, 052702 (2007).

S. D. Baton, M. Koenig, P. Guillou, et al., High Energy Density Physics 3, 358 (2007).

R. Kodama, H. Azechi, H. Fujita, et al., Nuclear Fusion, S276 (2004).

R. Kodama, P. A. Norreys, K. Mima, et al., Nature 412, 798 (2001).

R. Kodama, P. A. Norreys, Y. Sentoku, and R. B. Campbell, Fusion Science and Technology 49, 316 (2006).

R. J. Mason, Physical Review Letters 96, 035001 (2006).

T. Nakamura, H. Sakagami, T. Johzaki, H. Nagatomo, and K. Mima, Journal de Physique IV (Proceedings) 133, 401 (2006).

T. Nakamura, H. Sakagami, T. Johzaki, H. Nagatomo, K. Mima, and J. Koga, Physics of Plasmas 14, 103105 (2007).

M. Nakatsutsumi, R. Kodama, P. A. Norreys, et al., Physics of Plasmas 14, 050701 (2007).

Y. Sentoku, K. Mima, H. Ruhl, Y. Toyama, R. Kodama, and T. E. Cowan, Physics of Plasmas 11, 3083 (2004).

J. A. Koch, Y. Aglitskiy, C. Brown, et al., Review of Scientific Instruments 74, 2130 (2003).

H. Habara, K. Adumi, T. Yabuuchi, et al., Physical Review Letters 97, 095004 (2006).

Y. T. Li, X. H. Yuan, M. H. Xu, et al., Physical Review Letters 96, 165003 (2006).

T. Yabuuchi, K. Adumi, H. Habara, et al., Physics of Plasmas 14, 040706 (2007).

Y. Ping, R. L. Shepherd, B. F. Lasinski, et al., Physical Review Letters submitted for publication (2007).

S. Baton, private communication (2007).

M. Tabak, private communication (2007). 


\section{Figure Captions}

Figure 1. (a) $0.5 \mathrm{~mm}$ square, $25 \mu \mathrm{m} \mathrm{Cu}$ foil irradiated by the laser at $28^{\circ}$ with respect to the normal. (b) identical target irradiated at $75^{\circ}$. The laser direction is right to left in this figure. The intensity scale in (a) is a factor of 10 greater than in (b).

Figure 2. $\mathrm{Cu} \mathrm{K}$ 品 fluorescence for varied laser focusing conditions. The top row shows spatially resolved images of the fluorescence for the defocusing conditions illustrated in the schematic diagrams in the middle row. The bottom row shows axial lineouts along the center line of the cone image averaged over several pixels.

Figure 3. Axial lineouts from Figure 2 overlayed and normalized to the peak emission to show overall similarity.

Figure 4. Ray tracing results for the case of the laser focusing $400 \mu \mathrm{m}$ beyond the cone tip. The solid black lines show the typical ray path and the expanded region shows the $100 \mu \mathrm{m}$ nearest the cone tip. For these rays the angle of incidence with respect to the cone wall becomes less than about $45^{0}$.

Figure 5. Calculation from optical ray tracing including wall absorption showing the fraction of absorbed laser energy along the length of the cone. Note that significant absorption only occurs within about $50 \mu \mathrm{m}$ of the cone tip in the absence of preplasma. 


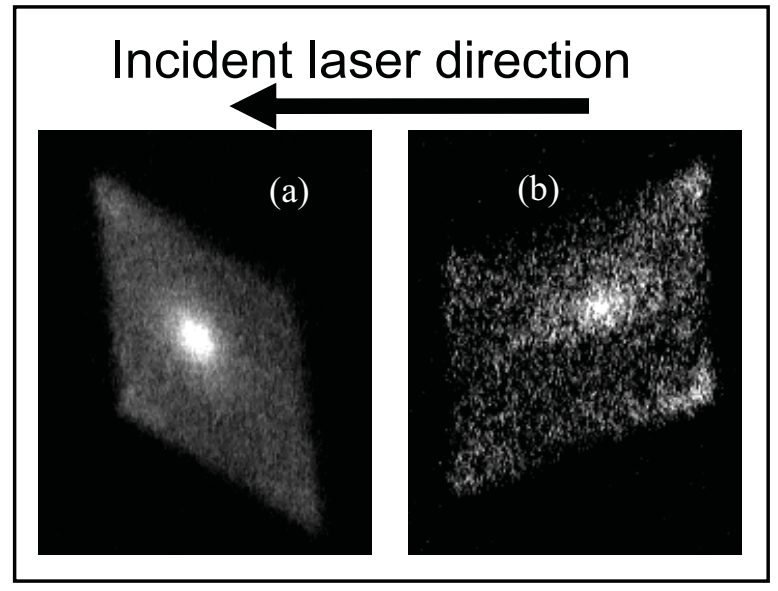




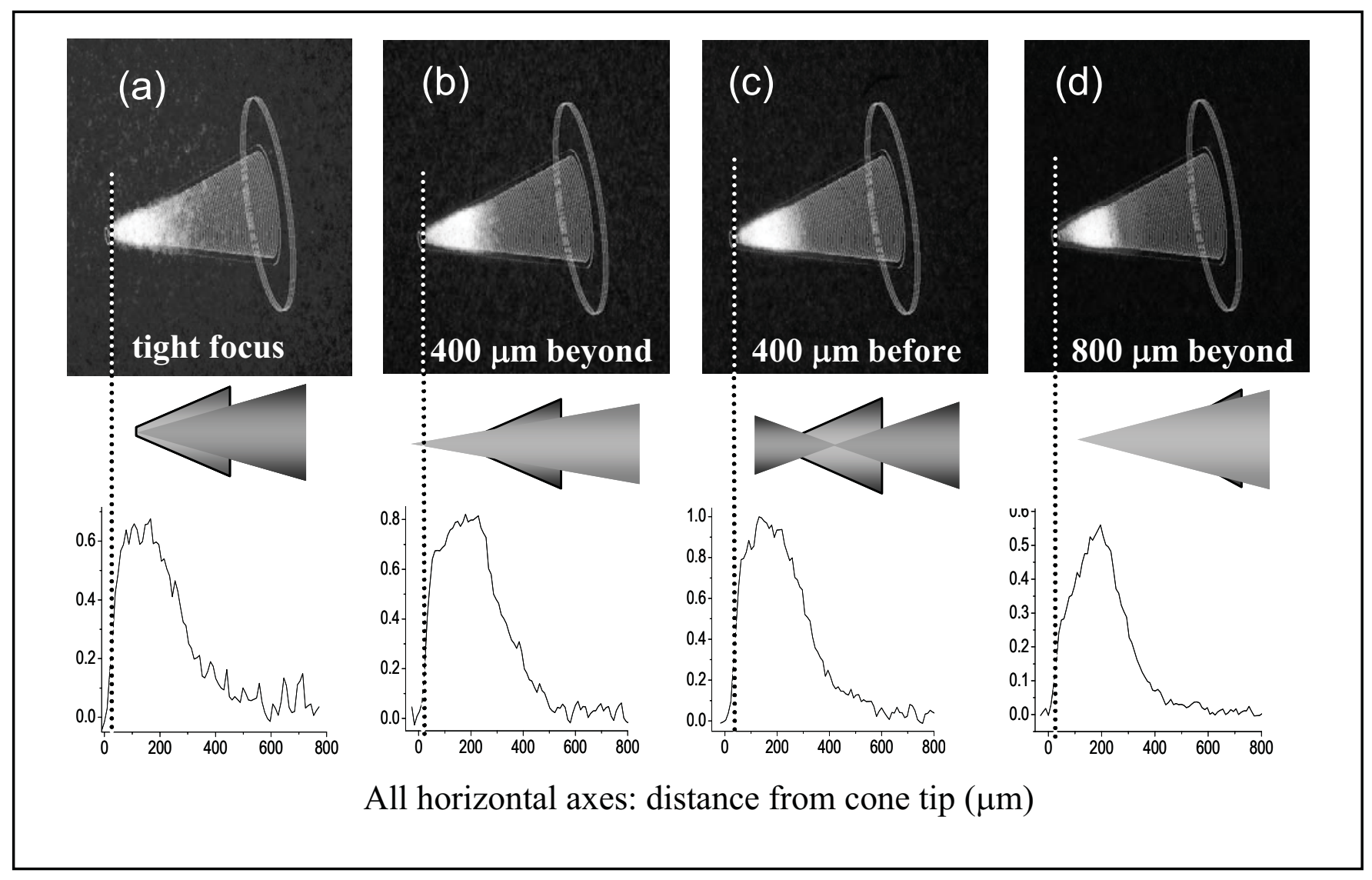




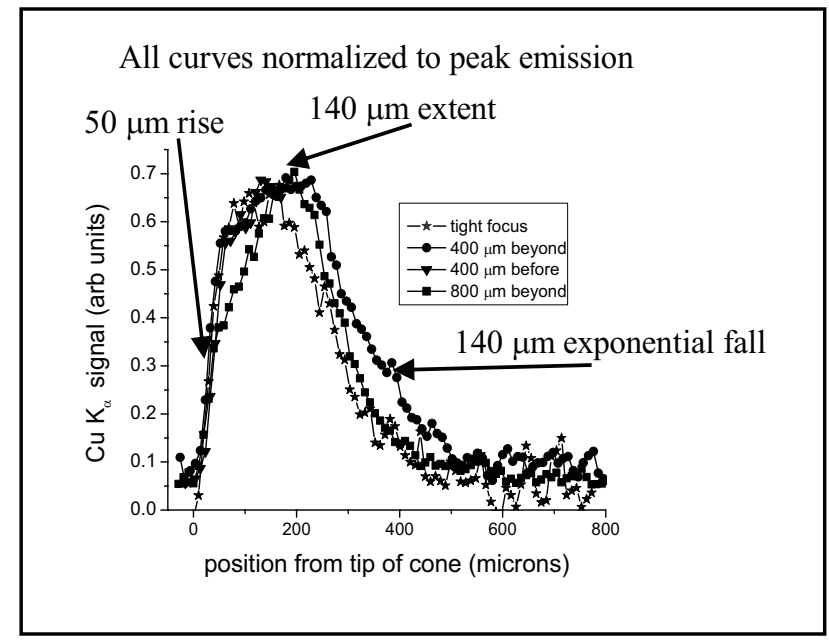




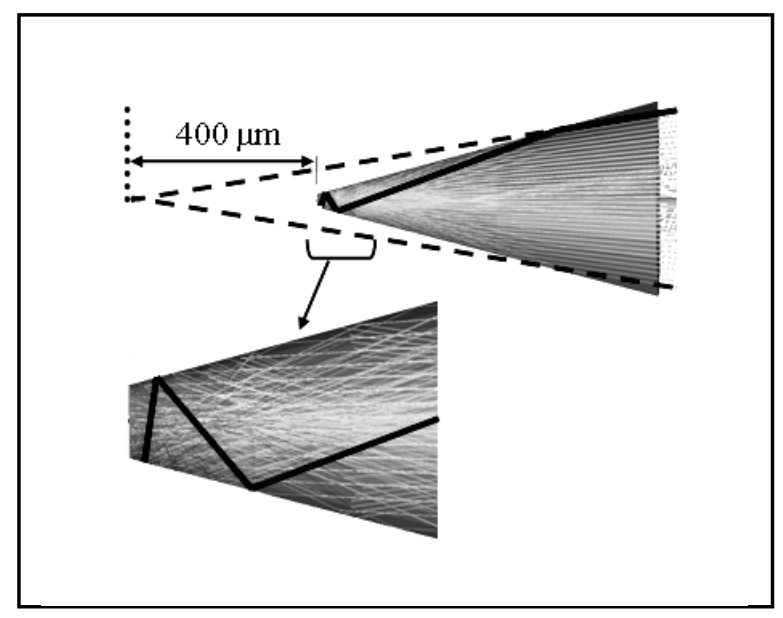




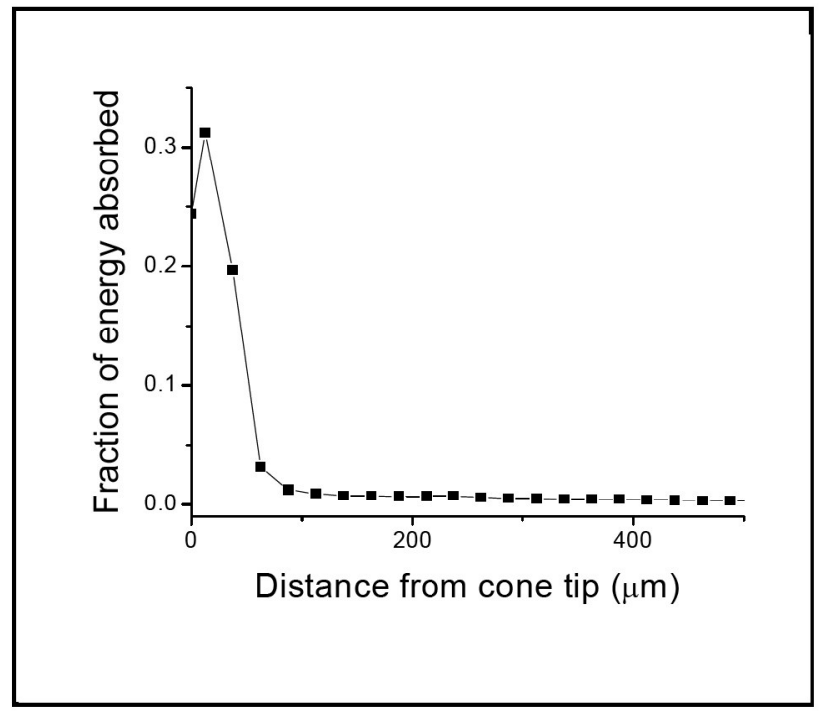

